\title{
Conocimientos, actitudes, prácticas y subregistro de dengue en el municipio de Tocaima, Cundinamarca, 2019.
}

\author{
Oscar Pacheco $\mathrm{G}^{1,2}$, Laura Trujillo $V^{2}$, Erika Delgadillo $\mathrm{C}^{2 *}$, Carolina Romero $\mathrm{R}^{2}$, Erika Gutiérrez D2, Yuli Salinas \\ $\mathrm{T}^{2}$, Rodrigo Merlano $\mathrm{A}^{2}$, Alba Sarria $\mathrm{R}^{2}$, Angelica Mora $\mathrm{C}^{2}$, Claudia Varela $\mathrm{A}^{2}$, Marcela González $\mathrm{T}^{2}$, Hermes Pérez \\ $\mathrm{B}^{2}$, Luis Gómez M2. Silvia Rodríguez $\mathrm{V}^{2}$, Iván Buitrago L², Bibiana Ávila B², Rafael Camacho C², Jorge Sotelo $\mathrm{S}^{2}$.

\section{RESUMEN}

Introducción. Pese a las estrategias de vigilancia se cree que existe Subregistro de dengue, lo que puede ser secundario a la no detección de casos ya sea por no consulta del paciente, por falla en el diagnóstico o diligenciamiento de fichas de notificación.

Objetivo. Estimar el Subregistro de los casos de dengue en el municipio de Tocaima durante el periodo de enero a septiembre de 2019 y establecer los conocimientos, actitudes y prácticas respecto al dengue de los habitantes del municipio.

Metodología. Se realizó un estudio transversal de prevalencia durante el período de enero a septiembre de 2019 en la población urbana de Tocaima, mediante encuestas a dos viviendas de las 262 manzanas seleccionadas por muestreo aleatorio simple en la búsqueda activa comunitaria y en el $100 \%$ de los registros individuales de prestación de servicios y del sistema de vigilancia nacional en salud pública SIVIGILA mediante Búsqueda Activa Institucional BAI.

Resultados. Se encuestaron 440 personas de las cuales 19 manifestaron haber presentado síntomas y signos de dengue en el periodo de estudio, de estos, seis personas no asistieron a consulta médica. El Subregistro Comunitario por falta de asistencia a consulta médica fue de $31,5 \%$. Se identificaron en SIVIGILA 125 casos de dengue notificados en el periodo y 156 en el Registro Individual de Prestación de Servicios (RIPS), aplicando el método de Chandra-Sekar Deming se estimaron un total de 257 casos, para un Subregistro Institucional de SIVIGILA de 48,5\%.

Conclusiones. Son diversas las razones por las cuales no se notificaron todos los casos de la enfermedad al sistema de vigilancia, la primera es que solo se notifican los casos de las personas que consultan a los servicios de salud y que además viven en Tocaima, la segunda es que algunos casos detectados no se notificaron al sistema de vigilancia por errores de procedimiento, o por desconocimiento del diagnóstico por el personal de salud.

Palabras clave: Dengue, Subregistro Comunitario, Subregistro Institucional, Aedes aegypti, Vigilancia Epidemiológica.

\section{ABSTRACT}

Knowledge, acttitudes, practices and dengue under registration Tocaima, Cundinamarca 2019.

Introduction. Despite the surveillance strategies, it is believed that there is an underreporting of dengue, which may be secondary to the non-detection of cases, either due to non-consultation of the patient, due to failure in the diagnosis or filling in of notification sheets.

Objective. Estimate the under-registration of dengue cases in the municipality of Tocaima during the period from January to September 2019 and to establish the knowledge, attitudes and practices regarding dengue of the inhabitants of the municipality

Methodology and Materials. A cross-sectional study of prevalence was carried out during the period from January to September 2019, in the urban population of Tocaima, through surveys of 2 dwellings of the 262 blocks selected by simple random sampling in the active community search, and in $100 \%$ of individual records of service provision and Sivigila through institutional active search.

Results. 440 people were surveyed, 19 of whom reported having symptoms and signs of dengue in the study period, of these, six people did not attend a medical consultation. The Community Underregistration for lack of attendance at medical consultation was $31.5 \%$. 125 cases of dengue reported in the period were identified in SIVIGILA and 156 in the Individual Service Provision Registry (RIPS), applying the Chandra-Sekar Deming method, a total of 257 cases were estimated, for a SIVIGILA Institutional Sub-registry of 48,5\%.

Conclusions. There are several reasons why not all cases of the disease were notified to the surveillance system, the first is that only the cases of people who consult the health services and who also live in Tocaima, La Second, some cases detected were not notified to the surveillance system due to procedural errors, or due to lack of knowledge of the diagnosis by health personnel.

Keywords: Dengue, Community Under-registration, Institutional Under-registration, Aedes aegypti, Epidemiological alertness. \section{Erika Daniela Delgadillo C. erika-delgadillo@juanncorpas.edu.co}

*Autor de correspondencia:

Como citar: Pacheco García OE, Trü̈llo Vargas LL, Delgadillo Cañón ED, Romero Rangel AC, Gutiérrez Dueñas EJ, Salinas Torres YM, Merlano Álvarez RA, et al. Conocimientos, actitudes, prácticas y subregistro de dengue en el municipio de Tocaima, Cundinamarca, 2019. Revista Cuarzo 2020;26(2):11-17.

Recibido: 12 de octubre de 2020 Aceptado: 2 de diciembre de 2020 Publicado: 30 de diciembre de 2020 DOI: https://doi.org/10.26752/cuarzo.v26.n2.501 


\section{INTRODUCCIÓN}

$E^{1}$ 1 dengue es una enfermedad infecciosa de transmisión vectorial, producida por un virus de la familia Flaviviridae, del cual se han identificado hasta la fecha 4 serotipos y cuyo transmisor fundamental es el mosquito Aedes aegypti, es una enfermedad ampliamente distribuida con repercusión en la salud humana y gran impacto en la economía nacional y mundial (1). Se presenta en los climas tropicales y subtropicales, sobre todo en las zonas urbanas y semiurbanas (2). Los síntomas aparecen promedio de cuatro a siete días después de la picadura infectiva (3).

Las regiones más afectadas son las Américas, Asia Sudoriental y el Pacífico Occidental (4). La incidencia del dengue está en aumento, se estiman 390.000 .000 de infecciones por dengue cada año, de los cuales 96.000 .000 se manifiestan clínicamente, con grandes brotes desde el año 2016 (1). La Región de las Américas notificó más de 2.380 .000 casos ese año y 1032 muertes. En 2017 se notificaron 584.263 casos de dengue en las Américas, con reducción del $73 \%$ en relación con el año 2016 (4). En el año 2019 se observa un fuerte aumento de los casos de dengue en la Región de las Américas, con mayores tasas de incidencia en Brasil, Colombia, Honduras y Nicaragua (5). Se calcula que cada año son hospitalizadas 500.000 personas con dengue grave, cuya tasa de letalidad estimada es del 2,5\% (4).

En Colombia la incidencia de dengue ha sido fluctuante pasando de 664,5 casos por 100.000 habitantes en riesgo en el año $2010,474,6$ casos por 100.000 habitantes en riesgo en el año 2013 y 90,7 casos por 100.000 habitantes en el año 2017 (6) y aumentó nuevamente de casos con incidencia de 179,9 casos por 100.000 habitantes en riesgo en el año 2018 calculado para la semana epidemiológica 52 (5). En la semana epidemiológica 35 de 2019 se notificaron 2.534 casos probables de dengue, de estas el $66,2 \%$ de los casos proceden de los departamentos de Meta, Tolima, Huila, Santander, Norte de Santander, Cesar, Casanare, y Antioquia (7).

El municipio de Tocaima pertenece al departamento de Cundinamarca, se encuentra ubicado a 400 m.s.n.m. con características geográficas adecuadas para la diseminación del vector y desarrollo de la enfermedad, considerándose zona endémica de dengue (8). Entre el año 2014 y 2018 se presentaron 224 casos de dengue, para el año 2019 hasta el mes de julio se reportaron 84 casos, durante la semana 4, 9, 13 y 23 se observan períodos de epidemia (9).

Pese a las estrategias de notificación se cree que hay Subregistro de los casos de dengue, lo que puede ser secundario a la no detección de casos por no consulta del paciente o por falla en el diagnóstico o diligenciamiento de fichas de notificación. Esto puede afectar la toma de decisiones sobre el control de la enfermedad, el manejo de recursos y el planeamiento en la organización de los sistemas de salud para la atención médica. Por otro lado, siendo el dengue una enfermedad relacionada con factores ambientales, sociales y culturales, es indispensable identificar en las poblaciones endémicas la identificación de factores de riesgo en lo relacionado con el desarrollo del vector, la prevención de la enfermedad y sus conocimientos y prácticas sobre la misma.

Teniendo en cuenta lo anterior los objetivos de este trabajo fueron estimar el Subregistro de los casos de dengue en el Municipio de Tocaima durante el periodo de enero a septiembre de 2019 e identificar los conocimientos, actitudes y prácticas respecto al dengue de los habitantes del municipio de Tocaima.

\section{MATERIALES Y MÉTODOS}

\section{Tipo de estudio y población.}

Se realizó un estudio transversal de prevalencia durante el período de enero a septiembre de 2019, en la población urbana de Tocaima, mediante encuestas a dos viviendas de las 262 manzanas seleccionadas por muestreo aleatorio simple en la Búsqueda Activa Comunitaria, y en el $100 \%$ de los Registros Individuales de Prestación de Servicios y del SIVIGILA mediante Búsqueda Activa Institucional.

\section{Muestra.}

Para la Búsqueda Activa Comunitaria se visitó la totalidad de las 262 manzanas del Municipio. Se hizo un muestreo aleatorio simple de la población urbana total correspondiente a 11.148 con un nivel de confianza del $95 \%$ y un error esperado de $5 \%$, con prevalencia desconocida (50\%), obteniéndose un tamaño muestral de 371 personas, se estimó un $10 \%$ adicional para reposición, estableciendo un total de 409 personas a encuestar. Se acudió a dos viviendas aleatorias de cada manzana y se entrevistaron las personas que aceptaron la participación voluntaria (dos personas promedio por manzana visitada). No se excluyeron personas con discapacidad visual, auditiva o con alguna limitación establecida por la edad, sin embargo, durante la recolección de datos los datos no se presentaron casos. El cuestionario se diligenció de forma electrónica por medio de dispositivos móviles, siendo el entrevistador (estudiantes de la Especialización de Epidemiología-Juan N. Corpas) quienes realizaron las preguntas a las personas participantes (Anexo figura 1).

\section{Variables y nivel de medición.}

Las variables nominales incluyeron sexo, nivel educativo, estrato, régimen de afiliación, factores de riesgo en la vivienda asociado a la reproducción del Aedes aegypti, conocimientos sobre el dengue, actitudes respecto a la prevención del dengue, autopercepción del conocimiento de la enfermedad. Las variables de razón edad, número de casos reportados en SIVIGILA, número de registros en el RIPS, número de personas que manifestaron tener síntomas y signos de dengue, número de personas que manifestaron tener síntomas y signos y consultaron al médico, número de personas que manifestaron tener síntomas y signos y no consultaron al médico.

\section{Base de datos.}

Para la búsqueda comunitaria se diseñó y aplicó un formulario para las variables correspondientes a la vivienda y uno para las variables de personas mediante el programa Epi Info $7 \AA$, se aplicó de manera presencial a un habitante de cada una de las 
viviendas seleccionadas de acuerdo con el muestreo, que fuera mayor de edad y estuviese dispuesto a responder de manera voluntaria, donde el encuestador mediante el uso de dispositivos móviles diligenciaba el formulario de acuerdo a las respuestas aportadas por el encuestado.

Para la Búsqueda Activa Institucional se utilizó el módulo SIANIESP del SIVIGILA en el que se cargaron el $100 \%$ de los RIPS entregados por el Hospital Marco Felipe Afanador de Tocaima, se seleccionaron los registros correspondientes al diagnóstico de dengue A90 y A91 de acuerdo con el CIE - 10.

\section{Análisis estadístico.}

Los datos obtenidos se digitaron y analizaron en Epi - Info 7®, se organizaron en distribuciones de frecuencias expresadas como proporciones, para las variables cuantitativas de razón se calcularon medidas de tendencia central y prevalencia de punto. Se utilizaron las siguientes fórmulas para los cálculos:

\section{Subregistro Comunitario (SC):}

$S C=\frac{\text { Número de personas que informaron haber presentado síntomas y signos y NO consultaron }}{\text { Número total de personas que informaron tener síntomas y signos }} \times 100$

\section{Subregistro Institucional (SI):}

Se aplicó la metodología de estimación de la integridad de dos sistemas de vigilancia independientes establecido por ChandraSekar y Deming para estimar el número total de casos de dengue en el Municipio, $\mathrm{N}=(\mathrm{a}+\mathrm{c})(\mathrm{a}+\mathrm{b}) / \mathrm{a}($ tabla 1).

Tabla 1: Estimación del total de casos de dengue, Tocaima, enero septiembre 2019

\begin{tabular}{llll}
\hline \hline \multicolumn{3}{c}{ FUENTE } & \multicolumn{3}{c}{ BAI-RIPS } \\
\hline \hline SIVIGILA & SI & NO & TOTAL \\
SI & 76 & 49 & 125 \\
NO & 80 & 52 & 132 \\
\hline \hline & $\mathbf{1 5 6}$ & $\mathbf{1 0 1}$ & $\mathbf{2 5 7}$ \\
\hline \hline \multicolumn{4}{c}{$\begin{array}{c}\text { Fuente: RIPS - SIVIGILA ESE Hospital Marco Fidel Afanador, } \\
\text { Método de Captura Recaptura, Chandra-Sekar Deming. }\end{array}$}
\end{tabular}

Chandra Sekar y Deming han desarrollado un procedimiento estadístico para estimar el número de casos no notificados en dos sistemas de registro que recojan una misma información de manera independiente. Este método, denominado CapturaRecaptura, permite aproximarse al conocimiento de la incidencia de enfermedades a partir de dos o más fuentes de información de manera coste- efectiva. En nuestro medio se ha aplicado este método por diversos autores obteniendo una estimación de la incidencia. (10).

$$
\widehat{N}=\frac{\frac{n 1}{n 2}}{m}
$$

N1: Cantidad de sujetos captados en un primer muestreo.

N2: Cantidad de sujetos captados en un segundo muestreo.

M: Cantidad de sujetos captados en la primera y segunda vez.

\section{RESULTADOS}

En la Búsqueda Activa Institucional (BAI), sobre los RIPS suministrados, se identificaron casos de dengue residentes en los Municipios aledaños por lo que se filtró la búsqueda a los casos de dengue exclusivamente del Municipio de Tocaima. Encontrándose en SIVIGILA 125 casos de dengue, en RIPS 82 casos y en la Búsqueda Activa Institucional (BAI) (se incluyen municipios aledaños) se encontraron 156 casos de dengue.

Con base en la información del SIVIGILA (125 casos) y de la Búsqueda Activa Institucional (BAI) (156 registros) de la institución, se estimó un Subregistro Institucional del 48,63\% (IC95 \% 42,9-87,4).

En la Búsqueda Activa Comunitaria se incluyeron 262 manzanas de las cuales fueron efectivas 203 manzanas del casco urbano del Municipio de Tocaima, se descartaron 59 manzanas dado que se encontraban en situaciones como, terrenos baldíos, casa lotes sin habitantes o zonas en construcción de acuerdo con la cartografía del Instituto Geográfico Agustín Codazzi, adicionalmente se enumeró como manzana lugares como parques, centros vacaciones cementerio etc. Se encuestaron 440 personas tomando dos personas por manzana en promedio. De las personas encuestadas, 19 refieren haber presentado síntomas y signos de dengue en el último año; cabe aclarar que no hubo evidencia serológica o de historia clínica que lo sustentara, con base en el número de casos detectados en la búsqueda activa comunitaria, las personas que no asistieron a consulta médica fueron seis, se estimó un Subregistro Comunitario por no consultar al servicio médico de $31,5 \%$, de ellas solo dos declararon que fue por falta de accesibilidad al sistema, 13 asistieron al Servicio de Salud y recibieron tratamiento.

Teniendo en cuenta los resultados obtenidos para la pregunta en la cual se evaluaba el conocimiento de que síntomas produce el dengue, el $86,74 \%$ de las viviendas respondieron que fiebre y en un 55,56\% el dolor de cabeza. Respecto al lugar de reproducción del vector en el 70,97 \% de las viviendas encuestadas respondieron que en agua sucia y un $38,71 \%$ en agua limpia. El 87,46\% de las viviendas encuestadas establece que la picadura de zancudo es el principal mecanismo de trasmisión del dengue. Al evaluar la percepción en las viviendas encuestadas respecto a si en algún momento había recibido educación sobre dengue el 49,82\% respondió que no (tabla 2) el $73,48 \%$ de viviendas encuestadas reconoce que la enfermedad del dengue es grave, el 19,35\% considera que es medianamente grave, el $2,87 \%$ considera que no es grave y el $4,30 \%$ no respondió a la pregunta. 
Tabla 2: Educación sobre dengue en el municipio de Tocaima Cundinamarca 2019.

\begin{tabular}{rrr}
\hline \hline EDUCACIÓN & FRECUENCIA & \% \\
\hline \hline Si & 136 & $48,75 \%$ \\
$N o$ & 139 & $49,82 \%$ \\
Sin registro & 4 & $1,43 \%$ \\
\hline \hline Fuente: encuestas trabajo de campo Tocaima, Cundinamarca 2019.
\end{tabular}

En Tocaima de las 276 viviendas encuestadas más del $90 \%$ de las mismas cuentan con servicio de acueducto alcantarillado. A pesar de las limitaciones en el servicio de agua, se evidencia que del total de la muestra el $81.36 \%$ (227 viviendas) cuentan con el servicio de agua diariamente con algunas intermitencias.

Con relación a los depósitos para el manejo de agua encontramos mayoritariamente la presencia de tanques de depósito de agua en $77,78 \%$ correspondiente a 217 viviendas. De las 276 viviendas encuestadas, la estrategia más empleada con un porcentaje de 57,35\% para la limpieza de los depósitos de agua, es el enjuague, cepillado y uso de cloro. El porcentaje restante $36,01 \%$ no realiza la técnica recomendada por el Ministerio de Salud, mientras un 6, $09 \%$ no los limpian (tabla 3). El $87,10 \%$ realiza la eliminación de los residuos cada vez que pasa el carro de la basura, según el plan de recolección de la alcaldía municipal 3 veces a la semana normalmente. La frecuencia de limpieza de los depósitos de agua se realiza semanalmente en el $35,48 \%$ de las viviendas encuestadas, se identificaron viviendas en las cuales no se realiza limpieza de estos en 5,38\% (tabla 4).

Tabla 3: Limpieza de depósitos de agua, en Tocaima Cundinamarca 2019.

\begin{tabular}{rcr}
\hline ESTRATEGIA LIMPIEZA & FRECUENCIA & \% \\
\hline \hline Enjuaga, cepilla y usa cloro & 160 & $57,35 \%$ \\
Enjuaga y cepilla & 61 & $21,86 \%$ \\
No los limpia & 17 & $6,09 \%$ \\
Sin registro & 16 & $5,73 \%$ \\
Sólo enjuaga & 13 & $4,66 \%$ \\
Sólo cepilla & 12 & $4,30 \%$ \\
\hline \hline
\end{tabular}

Fuente: encuestas trabajo de campo Tocaima, Cundinamarca 2019.

Tabla 4: Limpieza de depósitos de agua, en Tocaima Cundinamarca 2019.

\begin{tabular}{|c|c|c|}
\hline $\begin{array}{l}\text { LIMPIEZA } \\
\text { DEPOSITO } \\
\end{array}$ & FRECUENCIA & PORCENTAJE \\
\hline Semanal & 99 & $35,48 \%$ \\
\hline Mensual & 75 & $26,88 \%$ \\
\hline Trimestral & 30 & $10,75 \%$ \\
\hline Bimensual & 22 & $7,89 \%$ \\
\hline Semestral & 21 & $7,53 \%$ \\
\hline Nunca & 15 & $5,38 \%$ \\
\hline Anual & 11 & $3,94 \%$ \\
\hline Sin registro & 3 & $1,08 \%$ \\
\hline No hay & 3 & $1,08 \%$ \\
\hline
\end{tabular}

Las medidas de prevención más utilizadas en las viviendas encuestadas para prevenir el crecimiento del vector es la fumigación con un 51,25\% y la limpieza de los patios con 50,90 $\%$ (tabla 5). Entre los factores de riesgo indagados que puedan favorecer la presencia de la enfermedad encontramos con mayor frecuencia la presencia de bebederos de mascotas 37,28 $\%$ y pilas de almacenamiento $22.58 \%$ (tabla 5 ).

Tabla 5: Uso de medidas de protección para dengue en Tocaima -Cundinamarca 2019.

\begin{tabular}{llcl}
\hline \multicolumn{1}{c}{$\begin{array}{c}\text { Uso de alguna } \\
\text { medida }\end{array}$} & SI/NO & FRECUENCIA & $\%$ \\
\hline \hline $\begin{array}{l}\text { Colocar boca abajo } \\
\text { recipientes vacíos }\end{array}$ & $\mathrm{No}$ & 215 & $77,06 \%$ \\
Eliminación de & $\mathrm{Si}$ & 64 & $22,94 \%$ \\
aguas estancadas & $\mathrm{No}$ & 197 & $70,61 \%$ \\
& $\mathrm{Si}$ & 82 & $29,39 \%$ \\
Limpieza patios & $\mathrm{No}$ & 137 & $49,10 \%$ \\
& $\mathrm{Si}$ & 142 & $50,90 \%$ \\
Tapar reservorios de & $\mathrm{No}$ & 196 & $70,25 \%$ \\
agua & $\mathrm{Si}$ & 83 & $29,75 \%$ \\
& $\mathrm{No}$ & 215 & $77,06 \%$ \\
Toldillos & $\mathrm{Si}$ & 64 & $22,94 \%$ \\
& $\mathrm{No}$ & 198 & $70,97 \%$ \\
Repelentes & $\mathrm{Si}$ & 81 & $29,03 \%$ \\
& $\mathrm{No}$ & 136 & $48,75 \%$ \\
Fumigación & $\mathrm{Si}$ & 143 & $51,25 \%$ \\
Ropa de manga & $\mathrm{No}$ & 267 & $95,70 \%$ \\
larga & $\mathrm{Si}$ & 12 & $4,30 \%$ \\
\hline \hline
\end{tabular}

\section{DISCUSIÓN}

Al realizar una Búsqueda Activa Institucional y Comunitaria de dengue con base en la información del SIVIGILA (125 casos) y de la BAI (156 registros), se estimó un Subregistro Institucional del $51 \%$ (IC $95 \% 42,9-87,4$ ), cifra porcentual elevada que en Colombia y en estudios hechos en Latinoamérica ya ha sido similarmente documentada. El Subregistro de patologías de transmisión por vectores ha sido significativo por diferentes causas como las registradas en el estudio de la Secretaría Departamental de Salud de Santander, en el año 2010 donde revisaron 4.774 RIPS, encontrando registros con diagnóstico diferencial compatible con dengue, que cumplían los criterios de caso probable establecidos en el protocolo y no se reportaron al SIVIGILA, presentándose un Subregistro del 56\% (11), o en la estimación de Subregistro de casos de Chikunguña en Villeta Cundinamarca en 2015 donde la Búsqueda Activa Institucional realizada se encontraron casos con otros diagnósticos que llevan a cumplir definición de caso (85\%) (12).

Con base en el número de casos detectados en la Búsqueda Activa Comunitaria, las personas que no asistieron a consulta 
médica generaron un Subregistro Comunitario de 31,5 \%, y de estos quienes aseguraron no tuvieron acceso a los servicios médicos representaron el $10,52 \%$. Información que se asemeja al estudio de Estimación del Subregistro de Casos de la Enfermedad por el Virus de Chikunguña en el Municipio de Villeta, Cundinamarca en el 2014 donde se evidencia que el Subregistro se da porque las personas no consultaron al servicio médico siendo un Subregistro del 34,7\%, el principal motivo fue la automedicación en el $45,2 \%$ de los casos (12), en el Sistema de Vigilancia para la detección de casos de dengue en Belo Horizonte, Brasil en un periodo de 1992 a 2006 se encontró un Subregistro general del 37\% de casos que cumplían con los criterios establecidos, que al no representar la totalidad de los casos hospitalizados se sobreestima la Tasa de Mortalidad de la Enfermedad (13).

Con el fin de tener un acercamiento a las actitudes y prácticas que tienen los habitantes de la comunidad de Tocaima con respecto al dengue, se abordaron aspectos de conocimiento respecto al mecanismo de trasmisión, así como las prácticas de prevención en hogar. Se encontró que el 73,48 \% de los habitantes están de acuerdo que el dengue es muy grave, pudiendo llegar a ocasionar hasta la muerte, con respecto a las prácticas evidenciadas en la población estudiada se encuentra que tienen una baja adherencia a la información impartida por las entidades de salud frente a la forma de almacenar el agua, mantener recipientes secos, tapados y al aseo de estos reservorios de agua, la comunidad conoce que la transmisión del dengue es por medio de un zancudo que sirve como vector (87,46 \%). El estudio de Conocimientos, Prácticas y Aspectos Entomológicos del dengue en Medellín, Colombia es un estudio comparativo entre barrios con alta y baja incidencia, encontró que se ha recibido información respecto al dengue en menos de la mitad de la población (48,75\%), Según el análisis estadístico, en los barrios de baja incidencia, los participantes mostraron un mayor nivel de educación, Cerca del $50 \%$ de los participantes en los dos tipos de barrios tenía un conocimiento adecuado del vector, pues identificaron correctamente los sitios de cría y la relación de las larvas con el mosquito adulto. La situación fue similar en cuanto a los conocimientos sobre la transmisión y los síntomas (14). Esto nos refleja que el poseer conocimientos acerca de un tema, no garantiza unas prácticas preventivas, ni una actitud favorable para la prevención y control del dengue, así como se ve reflejado en los resultados, una parte de la población concuerda en haber recibido información, pero lo encontrado en las viviendas es totalmente diferente, ya que persiste el uso de inservibles y depósitos de aguas estancadas. El municipio de Tocaima presenta varias características o factores que determinan la presencia del evento, como, por ejemplo; la medida de prevención más utilizada es la fumigación, y en el control del vector la aplicación de sólo una estrategia no garantiza la eliminación permanente del problema vectorial; esto implica combinar adecuadamente diferentes mecanismos de tal forma que se garantice la permanencia de las acciones de control (15), por el contrario, hay un bajo porcentaje de la población que utiliza medidas como toldillos, uso de ropa manga larga o uso de repelente y eliminación de aguas estancadas, que son más efectivas . Para lo anterior, es indispensable vincular efectivamente a la comunidad en la implementación de medidas de control, de manera que se logre el control del vector en el ámbito domiciliario y se logre la realización de acciones de ordenamiento del ambiente cada vez que la población identifique factores de riesgo dado que los cambios sociales y demográficos, así como el crecimiento de la población, la urbanización sin planeación, el hacinamiento en los centros urbanos y rurales constituyen factores que propician la presencia de casos de dengue.

\section{CONCLUSIONES}

Son diversas las razones por las cuales no se notificaron todos los casos de la enfermedad al Sistema de Vigilancia, la primera es que solo se notifican los casos de las personas que consultan a los Servicios de Salud y que además viven en Tocaima, la segunda es que algunos casos detectados no se notificaron al Sistema de Vigilancia por errores de procedimiento, o por desconocimiento del diagnóstico por el personal de salud.

De las condiciones más relevantes para prevenir la aparición, transmisión de una enfermedad es tener el conocimiento de esta con el fin de poder implementar medidas que ayuden a solventar dicha situación, por eso es relevante generar medidas de educación que abarque la totalidad de la población. El control del dengue no es puramente del sector salud, se necesita de la intersectorialidad para que se limite la utilización de insecticidas o se realicen campañas de limpieza, modificando los factores sociales y culturales que favorecen la transmisión; pues estos se encuentran predominantemente en el ámbito familiar, ya que dependen íntimamente de la forma de vida de cada familia.

Recomendaciones: La percepción de educación brindada sobre el dengue está dividida, un 49,82 \% considera que no hay actividad educativa sobre el dengue en Tocaima, se midieron aspectos importantes como que los síntomas del dengue más ignorados por la población de Tocaima son dolor abdominal, dolor retro ocular en más del $90 \%$, y en la mayoría de las viviendas encuestadas alguna persona considera el agua sucia como lugar de reproducción del vector, es por eso que la Entidad Territorial de Salud debe garantizar la ejecución de las actividades de Vigilancia y Control de manera permanente y rutinaria, sin distinción de sexo, ciclo de vida, nivel socioeconómico o cualquier otra situación, para lo cual se recomienda la reorganización y el mejoramiento de los Programas de Promoción, Prevención y Control de las enfermedades transmitidas por vectores en el Municipio.

Las Secretarías Municipales de Salud deben hacer seguimiento continuo del cumplimiento de los lineamientos nacionales y de la Búsqueda Activa Institucional en todas las instituciones prestadoras de Servicios de Salud, para mejorar la notificación al Sistema de Vigilancia en Salud Pública del Municipio, en cumplimiento de la estrategia nacional de fortalecimiento de la autoridad sanitaria para la gestión de la salud, adicionalmente es importante que el personal de salud que labora en las instituciones prestadoras de servicios de salud se sensibilice frente a la importancia del reporte a los sistemas de información 
en salud en cumplimiento del Decreto 3518 del 2006.

Agradecimientos: Agradecemos al Municipio de Tocaima en cabeza del Alcalde Guilmar Alexander Martínez Bareño y su equipo de trabajo, al Doctor Carlos Andrés Prada Gerente del Hospital Marco Felipe Afanador por permitir que los Estudiantes de la Especialización en Epidemiología llevarán a cabo el estudio "Conocimientos, actitudes, prácticas y subregistro del dengue en el Municipio de Tocaima" y a su comunidad por el ingreso a sus hogares brindando información relevante para el mismo.

A la Fundación Universitaria juan N. Corpas por el conocimiento brindado y el acompañamiento durante el proceso de construcción y desarrollo del estudio.

Conflicto de Interés: Los investigadores declaran no tener ningún conflicto de interés en la realización del estudio.

\section{REFERENCIAS}

1. Palú A, García R, Tercero Méndez D, Torres M, Estrada L. características del virus del dengue y su pronóstico bioclimático en el departamento de cortés. medisan [internet]. 2017 sep. [citado 2019 nov 21] ; 21( 9 ): 2002$2009 . \quad$ disponible en: http://scielo.sld.cu/scielo.php?script=sci_arttext\&pid=s102 9-30192017000900005\&lng=es

2. Organización mundial de la salud, Nota descriptiva dengue, Fecha de consulta: 26 febrero de 2017 [citado 2019 nov 21]. disponible en: http://www.who.int/es/newsroom/factsheets/detail/dengue-and-severe-dengue.

3. Brady O, Gething P, Bhatt S, Messina JP, Brownstein JS, Hoen AG et al. Refining the global spatial limits of dengue virus transmission by evidence-based consensus. plos negl trop dis. 2012 [citado 2019 nov 21];6:e1760. doi:10.1371/journal.pntd.0001760.

4. Organización mundial de la salud. Nota descriptiva dengue. Fecha de consulta: 26 febrero de 2017 [citado 2019 nov 21]. disponible en: https://www.who.int/topics/dengue/es/

5. PAHO, Dengue en las américas. programa regional de dengue. septiembre de 2007. fecha de consulta: 21 sep de 2019. disponible

en: https://www.paho.org/hq/index.php?option=com_content\& view=article\&id=2433:2010-actualizacion-regionaldengue-programa-regional-dengue-04-febrero2010\&itemid $=42346 \&$ lang $=$ en

6. OPS, Diagnóstico dengue. [citado 2019 nov 21] disponible en:https://www.paho.org/hq/index.php?option=com_conten t\&view=article\&id=4493:2010-informacion-generaldengue $\&$ itemid $=40232 \&$ lang $=$ es.

7. Plan decenal de salud pública 2012-2021,Ministerios de salud y Protecciòn social de Colombia, fecha de consulta: 21 sep de 2019. disponible en: https://www.minsalud.gov.co/documentos $\% 20 \mathrm{y} \% 20$ publica ciones/plan $\% 20$ decenal $\% 20$ -

$\% 20$ documento $\% 20$ en $\% 20$ consulta $\% 20$ para $\% 20$ aprobaci $\%$ c3\%b3n.pdf

8. SIVIGILA, Situación del dengue, virus del zika y chikungunya en colombia, Fecha de consulta: 21 sep de $2019 . \quad$ disponible en: https:/temas.sld.cu/vigilanciaensalud/2019/09/12/situaciondel-dengue-virus-del-zika-y-chikungunya-en-colombia/

9. Instituto Nacional de Salud Colombia, Protocolo de vigilancia en salud pública, fecha de consulta: 21 sep de $2019 . \quad$ disponible en https://www.ins.gov.co/noticias/dengue/7.\%20dengue $\% 20 p$ rotocolo.pdf

10. Chandra Sekar C, Edwards Deming W. On a method of estimating birth and death rates and the extent of registration. Journal of the American statistical Association. 1949:44:101-15. doi: 10.1590/1980-5497201700020002.

11. Secretaría de Salud de Santander, Castañeda, Segura, Ramírez, Conocimientos, actitudes y prácticas comunitarias en un brote de Dengue en un municipio de Colombia, Bucaramanga, Colombia, 2010.

12. Pacheco O, Martínez M, Alarcón A, Bonilla M, Caycedo A, Valbuena T, Zabaleta A, Estimación de subregistro de casos de Chikunguña en Villeta Cundinamarca en 2015, Revista Biomédica, Vol. 37 Núm. 4 (2017), [Internet], Disponible: https://doi.org/10.7705/biomedica.v37i4.3370

13. Duarte H, Pelluci, H França E,. Qualidade dos dados da vigilância epidemiológica da dengue em Belo Horizonte, MG. Rev. Saúde Pública [Internet]. 2006 Feb [cited 2019 June 14]; 40(1): 134-142. Available from: http:// www.scielo.br/scielo.php?script=sci_arttext\&pid=S0034$89102006000100021 \& \operatorname{lng}=$ en http://dx.doi.org/10.1590/S0034- 89102006000100021

14. Ortiz C, Rúa-Uribe1 G, Rojas C, Conocimientos, prácticas $\mathrm{y}$ aspectos entomológicos del dengue en Medellín, Colombia: un estudio comparativo entre barrios con alta y baja incidencia, Revista Biomédica 2017; [Internet] 106-16 citada Junio 2020, doi:

15. Instituto nacional de salud, Subdirección de Vigilancia y Control en Salud Pública Protocolo de vigilancia y control de Dengue INT- PRO-R02.003.0000-004. Disponible en https://www.paho.org/col/index.php?option=com_docman \&view=download\&category_slug=publicaciones-ops-omscolombia\&alias $=1216$-protocolo-para-la-vigilancia-ensalud-publica-del-dengue $\&$ Itemid $=688$ 
Anexo Figura 1. Formulario

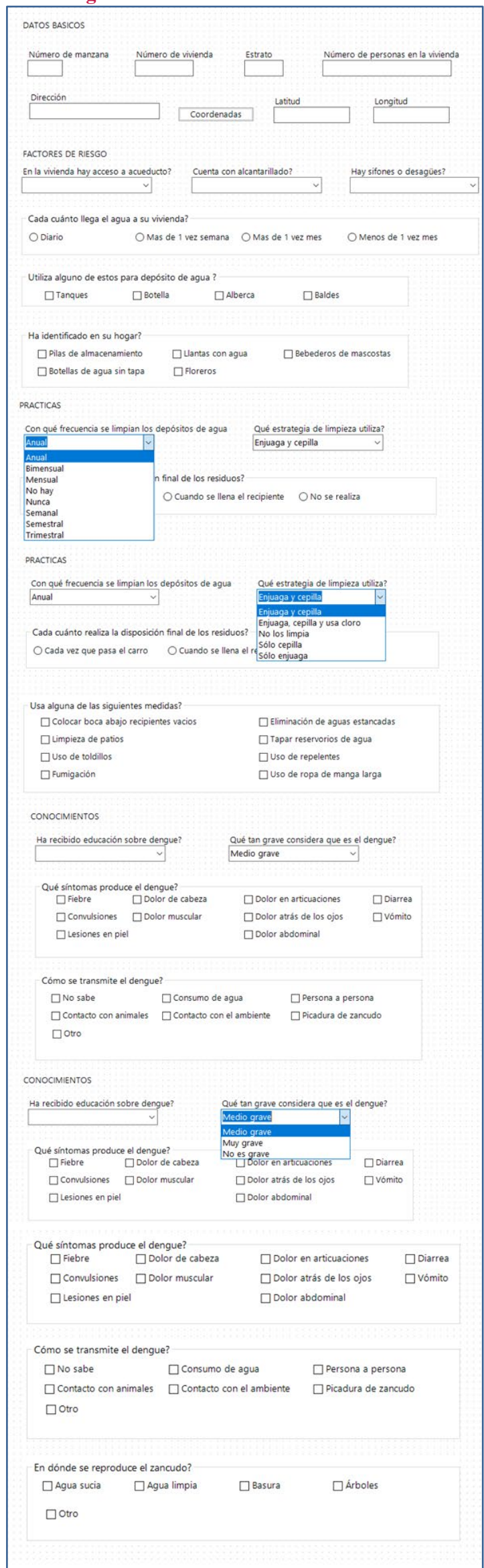

